\title{
A aplicação do modelo FPSEEA no gerenciamento de resíduos de serviço de saúde
}

\author{
Application of the DPSEEA Model to Healthcare \\ Waste Management
}

Nilva Lúcia Rech Stedile ${ }^{1}$

Vania Elisabete Schneider ${ }^{1}$

Monique Walltrick Nunes ${ }^{1}$

Adriane Carine Kappes ${ }^{1}$
${ }^{1}$ Mestrado Profissional em Engenharia e Ciência Ambientais, Centro de Ciências da Vida, Universidade de Caxias do Sul. R. Francisco Getúlio Vargas 1130, Petrópolis. 95001-970 Caxias do Sul RS Brasil.nrlstedi@ucs.br
Abstract The use of health indicators is indispensable for understanding the complex relationship between the environmental and health fields. For the proposition of environmental health indicators, the Brazilian Ministry of Health (MH) recommends using a model proposed by the World Health Organization. This model is composed of the following elements: Driving Force, Pressure, State, Exposure, Effect, and Action; it is called the DPSEEA model. The objective of this study is to propose the application of this model in healthcare waste (HCW) management. This study was performed by documentary research using two data sources: a) technical reports on research on HCW from the last 15 years (outpatient, dental, hospital, veterinary, university, and primary care unit) at the University of Caxias do Sul, Brazil; and $b$ ) the MH manual entitled "Environmental Health: Basic Guide for the Construction of Indicators". The results show that the model is relevant because it makes it possible to analyse a particular context, proposing indicators and defining specific actions for the case of this study, to monitor and improve methods of managing HCW. The results also show that the proposed model is an important analytical tool for both medical waste management and planning actions that will minimize risks, particularly chemical and biological, resulting in environmental health and protection.

Key words Environmental health, Health indicators, Healthcare waste
Resumo Para compreender a complexa relação entre o campo ambiental e o da saúde é indispensável o uso de indicadores. Para propor os de Saúde Ambiental, o Ministério da Saúde recomenda um Modelo proposto pela OMS. Este, denominado Modelo FPSEEA, é composto pelos elementos: Força Motriz, Pressão, Situação, Exposição, Efeito, Ações. O objetivo deste artigo é propor a aplicação deste Modelo no Gerenciamento dos Resíduos de Serviço de Saúde (RSS). Trata-se de pesquisa documental utilizando duas fontes de dados: a) relatórios técnicos de pesquisas sobre RSS dos últimos 15 anos (ambulatorial, odontológico, hospitalar, veterinário, universitário e de UBS) na Universidade de Caxias do Sul; b) Manual do MS "Saúde Ambiental: guia básico para construção de indicadores". Os resultados evidenciam que o Modelo é relevante por permitir analisar determinado contexto, propor indicadores e definir ações específicas para, no caso deste estudo, monitorar e aprimorar formas de manejo dos RSS. Evidenciam, ainda, que o modelo proposto constitui uma importante ferramenta de análise, tanto na MWM, como no planejamento de ações que minimizem os riscos, especialmente biológicos e químicos, resultando em saúde e proteção do ambiente.

Palavras-chave Saúde ambiental, Indicadores de saúde, Resíduos de serviço de saúde 


\section{Introdução}

Os processos produtivos de trabalho e de consumo são fatores que influenciam o estado de saúde humana e de saúde ambiental. O Ministério da Saúde ${ }^{1}$, ao abordar o contexto da saúde ambiental, afirma que as formas de produção e de desenvolvimento social e econômico interferem nos ecossistemas, que são determinantes e contribuintes na alteração do padrão e dos níveis de saúde da população. Tal interferência resulta na alteração do perfil epidemiológico e do perfil de morbimortalidade, em função da exposição do homem a diferentes e alteradas situações ambientais.

Somado às interferências ambientais que produzem adoecimento há a forma como os serviços de saúde focam suas ações, com objetivo pontual na doença. A lógica do atendimento em saúde focado na fisiopatologia, centrado no modelo biomédico que prioriza tratamentos e procedimentos e as características de organização dos serviços, leva a essa tendência de aumento progressivo do uso de descartáveis e, por consequência, o aumento do volume de resíduos gerados. Os descartáveis têm maior aceitação no setor saúde em função da sua praticidade e por reduzirem o risco de transmissão de doenças, especialmente as veiculadas pelo sangue e secreções. Nesse sentido, a geração e o gerenciamento dos resíduos gerados na assistência à saúde podem gerar riscos, tanto aos envolvidos diretamente com o trabalho em saúde, como aos usuários, suas famílias e à sociedade como um todo, caso esses resíduos sejam dispostos inadequadamente no meio ambiente ${ }^{2}$. Constituem-se, portanto, em um problema de Saúde Ambiental.

A questão de saúde ambiental pode ser considerada recente. A partir da proposição do documento Subsídios Para a Construção da Política Nacional de Saúde Ambiental ${ }^{3}$, várias iniciativas, em diferentes áreas, têm buscado aproximar saúde e meio ambiente, na direção de entender a complexa rede de relação entre ambas.

Disso decorre também a busca pelo desenvolvimento da legislação cuja aplicação pode proteger o ambiente e a saúde. Exemplo disso é a Política Nacional de Resíduos Sólidos (PNRS), que classifica os resíduos em função da origem (domicílio, construção civil, indústria, serviço de saúde, entre outros) e quanto à periculosidade (perigosos ou não perigosos). Os Resíduos de Serviço de Saúde (RSS) são definidos nesta Política como "[...] os gerados nos serviços de saúde $[. .] "$.4 e são considerados perigosos por conterem características de "[...] inflamabilidade, corrosividade, reatividade, toxicidade, patogenicidade, carcinogenicidade, teratogenicidade, mutagenicidade $[\ldots]]^{\prime 3}$, por apresentarem riscos importantes à saúde pública e qualidade do meio ambiente, especialmente os pertencentes a categoria Infectantes (Grupo A), Químicos (Grupo B), Radioativos (Grupo C) e Perfurocortantes $(\text { Grupo E })^{5}$

A geração de resíduos de serviços de saúde (RSS), embora seja relativamente pequena em relação aos resíduos sólidos urbanos, torna-se representativa quando considerado o potencial de risco que lhes é associado, devido à presença de organismos patogênicos e/ou de suas toxinas, produtos químicos de natureza diversa (fármacos, quimioterápicos, solventes, entre outros), bem como riscos radiológicos. A problemática dos RSS é decorrente ainda de outros fatores, tais como a mistura de resíduos de natureza diversa, descartados em via pública e/ou a disposição inadequada em aterros, nem sempre sanitários ou controlados ${ }^{2}$

Estes riscos são aumentados quando as formas de manejo de resíduos não são adequadas. As inadequações no manejo dos resíduos na sociedade contemporânea comprometem a qualidade de vida da população e a qualidade do meio ambiente, do que resulta riscos à saúde física (doenças infecciosas, degenerativas), à saúde mental (crises de ansiedade, crises de pânico, depressão) e a desintegração social (isolamento social, exacerbação da violência, entre outros $)^{6}$. O gerenciamento inadequado destes resíduos pode expor a saúde de trabalhadores a riscos diretos e a população a riscos indiretos, por meio da ação dos mesmos no ambiente ${ }^{7,8}$.

Esses aspectos sinalizam a indissociável relação existente entre saúde, saúde ambiental, meio ambiente e resíduo de serviço de saúde (RSS), os quais podem produzir efeitos adversos sobre o ambiente e os organismos. Nessa direção, o Ministério da Saúde ressalta que "ambiente e saúde são interdependentes e inseparáveis"3.

O conceito de Saúde Ambiental vincula os aspectos que envolvem a saúde humana e a qualidade de vida, determinados por fatores físicos, químicos, biológicos, sociais e psicológicos no meio ambiente, bem como expõe fatores do meio ambiente que são potenciais para acometer o estado de saúde das gerações atuais e futuras?

Para contribuir com esclarecimentos sobre as relações entre saúde e ambiente, a área da saúde tem feito uso de indicadores, os quais contribuem para a proposição de estratégias de promoção à 
saúde, prevenção e controle de riscos. Um indicador é definido como a descoberta e/ou informação de um conjunto de dados sobre determinada realidade. São ferramentas para gestão e tomada de decisões. O Ministério da Saúde também o define como um modelo que simplifica a realidade com objetivo de compreender fenômenos e eventos, de maneira a potencializar a comunicação de dados brutos e harmonizar as informações à linguagem e interesses de diferentes atores sociais ${ }^{1}$.

Segundo a definição da Rede Interagencial de Informações para a Saúde (RIPSA) ${ }^{10}$, um indicador pode ser entendido como uma medida-síntese que possui informação de relevância a respeito do estado de saúde, como também do desempenho do sistema de saúde. Assim, o papel de um indicador é identificar tendências, destacar e priorizar ações frente aos problemas, bem como decisões para formular e avaliar políticas e programas. Também servem à simplificação de um conjunto complexo de dados que diz respeito aos aspectos da saúde e meio ambiente, o que contribui para melhor comunicação entre o setor público e os gestores ${ }^{10}$.

Existem indicadores específicos para cada área, como os de saúde e de meio ambiente, e aqueles relacionados a ambas, como os de saúde ambiental. Para o setor saúde são usados tradicionalmente os indicadores de mortalidade, de morbidade, de cobertura de serviços e atendimentos, entre outros. Para o setor ambiental e de saúde ambiental, os indicadores são mais recentes se comparados com a área social e da saúde, o que configura os esforços para aprimoramento e intersetorialidade entre áreas ${ }^{1}$.

Como recurso para elaboração de indicadores de saúde ambiental a OMS propôs o uso de um Modelo, o qual tem sido utilizado como ferramenta no gerenciamento ambiental em todo mundo, passando a ser recomendado também no Brasil pelo Ministério da Saúde. Desde então tem sido utilizado no âmbito dos governos e organizações não governamentais como estratégia de enfrentamento dos problemas ambientais decorrentes do modelo de desenvolvimento.

O Modelo inicial foi proposto pelo estatístico canadense Anthony Friend, na década de 70, sendo composto de três elementos básicos: pressão, estado e resposta. Aquele modelo foi denominado PER. No final da década de 90, este Modelo inicial foi ampliado pela OMS, constituindo-se em uma ferramenta para proposição de indicadores de Saúde ambiental, quando passou a ser constituído por seis elementos: Força motriz Pressão - Situação - Exposição - Efeito - Ação e denominado Modelo FPSEEA ou FPEEEA ${ }^{11}$. Em 2011, o Ministério da Saúde recomenda o uso do Modelo FPSEEA ${ }^{1}$ no Brasil.

O Objetivo do Modelo é fornecer um instrumento para o entendimento das relações abrangentes e complexas entre saúde e ambiente, permitindo analisar os problemas de Saúde Ambiental em toda sua cadeia causal. Possibilita compreender e mensurar os determinantes ambientais de saúde que contribuem para a nitidez dos processos decisórios voltados para o controle de riscos e formular indicadores que favorecem a integração dos Sistemas de Informação e a tomada de decisões por profissionais e gestores. Cabe destacar que a solução dos problemas de saúde pública relacionados às questões ambientais tem como pré-requisito a implementação de políticas públicas que criem e garantam a manutenção de ambientes saudáveis, a partir de processo participativos de gestão ${ }^{12}$. O Modelo possibilita a intersetorialidade, pois integra a informação de diversos setores, em especial a relação indissociável entre saúde e meio ambiente.

É com este foco que o MS e OPAS iniciaram em 1998 a implementação de uma agenda conjunta para construção de indicadores de saúde ambiental. Fizeram o uso pioneiro do Modelo FPSEEA no Brasil, o qual permitiu a construção de indicadores que foram base para implantação de importantes Programas de Vigilância em Saúde do País, como o Programa de Vigilância da Qualidade da Água para Consumo Humano (VIGIAGUA) e o Sistema de Informação para a Vigilância da Qualidade da Água para Consumo Humano (SISAGUA) na Secretaria de Vigilância em Saúde do Ministério da Saúde ${ }^{13}$.

O Modelo tem sido usado por diferentes autores, em diversos países e situações. Exemplo são estudos sobre os riscos do uso de agrotóxicos em atividades agrícolas ${ }^{14}$ na hantavirose, dengue, problemas respiratórios no Distrito Federal ${ }^{15}$, sobre determinantes socioambientais da saúde ${ }^{16}$; indicadores de saúde ambiental para região europeia (qualidade da água, qualidade do ar, condições de domicílio, acidentes de trânsito, ruído, resíduos e contaminação do solo, radiação, alimentos seguros, emergências químicas, locais de trabalho $)^{17}$; metodologias para a organização de indicadores de saúde ambiental em nível local e internacional da OMS (mortalidade infantil, resíduo domiciliar, pobreza, entre outros exemplos de aplicação) ${ }^{18}$; indicadores relacionados à saúde das crianças $^{19}$; formulação e avaliação de políticas de desenvolvimento sustentável ${ }^{13}$; riscos relacionados ao saneamento inadequado ${ }^{20}$. 
Cabe destacar que são nas causas de maior impacto desta cadeia (as forças motrizes, pressão, situação) que as intervenções são mais resolutivas e permitem que ocorra a integração do setor saúde com os demais, os quais são determinantes da saúde ambiental e que têm influência sobre as "causas das causas". Em outras palavras, são nesses âmbitos que a tomada de decisões políticas influencia a realidade da coletividade e a execução das ações realizada pelos profissionais.

Assim, este Modelo possibilita uma organização diferenciada dos indicadores de saúde ambiental, que objetiva mensurar e monitorar os possíveis agravos e riscos à saúde que são resultantes das interferências constantes e intensas das mudanças sociais, econômicas e ambientais ${ }^{1}$. $\mathrm{O}$ Modelo pode ser apresentado esquematicamente conforme Figura 1.

O objetivo deste artigo é aplicar o Modelo FPSEEA ao gerenciamento de resíduos de serviço de saúde, dado o impacto desses à Saúde Pública e Ambiental. Objetiva ainda levantar indicadores relacionados à temática.

\section{Método}

Este estudo se caracteriza como pesquisa documental. A pesquisa documental é definida como o método que utiliza materiais que não foram tratados analiticamente ou que podem ser reelaborados conforme os objetivos da pesquisa. Esta metodologia é embasada na exploração de documentos diversificados e dispersos, dentre os quais: os arquivos de órgãos públicos e privados, cartas pessoais, diários, fotografias, ofícios, boletins, entre outros ${ }^{21}$. Os documentos utilizados neste estudo estão apresentados no Quadro 1.

Os documentos foram lidos na íntegra, por meio de leitura flutuante, após foram selecionados os extratos de textos e dados relacionados aos objetivos deste estudo, os quais foram organizados em planilhas previamente construídas. O conjunto de dados sistematizados foi analisado, resultando na proposição de determinantes, indicadores e ações. Os extratos de textos foram sistematizados e organizados de acordo com os elementos do Modelo FPSEEA. A interpretação posterior seguiu os preceitos da análise de conteúdo ${ }^{22,23}$. Os resultados foram apresentados na forma de um Quadro síntese.

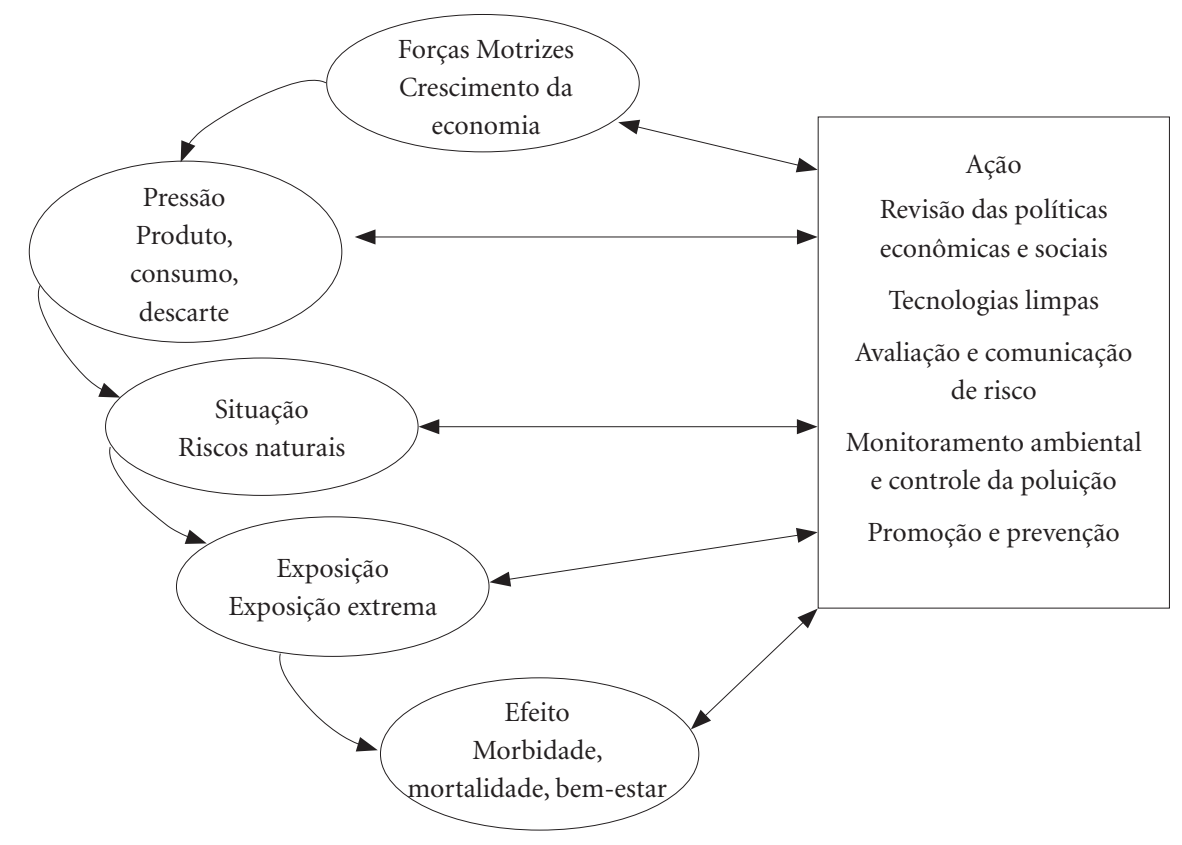

Figura 1. Modelo FPSEEA de construção de indicadores de saúde ambiental. 
Quadro 1. Documentos utilizados para compor o corpus deste estudo.

\begin{tabular}{|c|c|c|c|}
\hline Título do Documento & Ano & $\begin{array}{c}\text { Local de } \\
\text { Origem do } \\
\text { Documento }\end{array}$ & $\begin{array}{l}\text { Critérios de Seleção } \\
\text { do Documento }\end{array}$ \\
\hline $\begin{array}{l}\text { Manual de Saúde Ambiental: guia básico para proposição } \\
\text { de indicadores. }\end{array}$ & 2011 & $\begin{array}{l}\text { Ministério da } \\
\text { Saúde }\end{array}$ & $\begin{array}{l}\text { Este é o guia básico } \\
\text { para formulação de } \\
\text { indicadores de saúde } \\
\text { ambiental no Brasil. }\end{array}$ \\
\hline $\begin{array}{l}\text { Relatórios Técnicos das seguintes pesquisas: } \\
\text { - Sistematização de fontes geradoras de resíduos sólidos de } \\
\text { serviços de saúde } \\
\text { • Estudo da geração de resíduos sólidos odontológicos } \\
\text { (RSSO) no Município de Caxias do Sul como subsídio para } \\
\text { a formulação de políticas de gerenciamento } \\
\text { - Gerenciamento de Resíduos Sólidos em Unidades Básicas } \\
\text { de Saúde no Município de Caxias do Sul - Subsídios para a } \\
\text { formulação de modelos. } \\
\text { - Sistematização de RSS em Universidade } \\
\text { - Gerenciamento de RSS em hospital e ambulatório escola } \\
\text { - Gerenciamento de Resíduos nas Unidades de Assistência } \\
\text { e Pesquisa em Saúde na Universidade de Caxias do Sul: } \\
\text { Avaliação de cenários e perspectivas } \\
\text { - Resíduos de Serviços de Saúde: Impacto de um programa } \\
\text { de educação permanente sobre a segregação e minimização } \\
\text { da geração } \\
\text { - Sistematização de fontes geradoras de resíduos } \\
\text { veterinários: mapeamento com vistas à formulação de } \\
\text { modelos de gerenciamento } \\
\text { • Educação permanente e informações em saúde como } \\
\text { estratégias de fortalecimento da atenção básica. }\end{array}$ & $\begin{array}{l}2000 \\
2001 \\
2005 \\
2008 \\
2009 \\
2010 / \\
2011 \\
2013\end{array}$ & $\begin{array}{l}\text { ISAM/UCS } \\
\text { (Instituto de } \\
\text { Saneamento } \\
\text { Ambiental da } \\
\text { Universidade de } \\
\text { Caxias do Sul) }\end{array}$ & $\begin{array}{l}\text { Os relatórios técnicos } \\
\text { contêm dados } \\
\text { confiáveis sobre o } \\
\text { gerenciamento de } \\
\text { RSS em diferentes } \\
\text { fontes geradoras, } \\
\text { incluindo indicadores } \\
\text { de gerenciamento de } \\
\text { interesse neste estudo. }\end{array}$ \\
\hline
\end{tabular}

Fonte: elaborado pelos autores (2016).

As informações referentes aos RSS foram aplicadas ao Modelo FPSEEA, com base nas definições de seus elementos constituintes: a) força motriz, que corresponde aos fatores de escala macro nos vários processos que influenciam a saúde ambiental e humana (exemplo: a taxa de urbanização e taxa de crescimento populacional); b) pressão, que se expressa pelas consequências destes processos (exemplo: o consumo de energia); c) situação, que é o resultado da pressão no ambiente. A situação resulta no aumento da frequência ou magnitude de situações ambientais que afetam negativamente à saúde (exemplos: taxas $\mathrm{e}$ indicadores de seca, qualidade da água e ar); d) exposição, que é o resultado da situação que resultou da pressão e força motriz. É definida como conceito chave porque estabelece as relações das situações ambientais e seus efeitos sobre o estado de saúde da comunidade e população; e) efeito, que é o resultado da exposição sobre a saúde humana e ambiental, que pode se manifestar subclinicamente (pela redução do bem-estar) ou até como doenças e condições extremas (Exemplos: indicadores de morbimortalidade); f) ação, componente que leva a gestão e tomada de decisões sobre os problemas sistematizados. Essas ações devem ser realizadas pelo poder público, para formular e programar soluções para que meio ambiente e população possam viver e estar saudáveis para exercerem suas funções ${ }^{1}$.

\section{Resultados e discussão}

O cenário estudado é apresentado no Quadro 2, que mostra a aplicação do Modelo FPSEEA ao gerenciamento dos RSS. O Quadro não tem por objetivo esgotar o número de indicadores e de ações necessárias, mas demonstrar possibilidade do uso desta ferramenta na temática em estudo e seu alcance.

A abrangência do Quadro 2 mostra que a aplicação do Modelo FPSEEA é relevante para subsidiar a construção de indicadores e para 
Quadro 2. Aplicação do Modelo FPSEEA no gerenciamento dos resíduos de serviço de saúde.

\begin{tabular}{|c|c|c|c|}
\hline $\begin{array}{l}\text { Nível do } \\
\text { Modelo }\end{array}$ & Determinantes & Indicadores & Ações \\
\hline \multirow[t]{2}{*}{$\begin{array}{l}\text { Força } \\
\text { Motriz }\end{array}$} & $\begin{array}{l}\text { Modelo } \\
\text { econômico } \\
\text { vigente. }\end{array}$ & $\begin{array}{l}\text { - PIB per capita. } \\
\text { - Indicadores de desenvolvimento } \\
\text { sustentável. } \\
\text { - Taxa de urbanização e de crescimento } \\
\text { populacional. } \\
\text { - Taxa de frota de veículos. } \\
\text { - Taxa da qualidade do ar e da água. } \\
\text { - Taxa de emprego e de renda. } \\
\text { - Índice de aumento do uso de } \\
\text { descartáveis. }\end{array}$ & \multirow{2}{*}{$\begin{array}{l}\text {-Instituir e aplicar políticas públicas } \\
\text { para desenvolvimento sustentável. } \\
\text { - Implementar educação ambiental } \\
\text { a comunidades e em situação de } \\
\text { ensino formal. } \\
\text { - Reduzir a emissão de poluentes no } \\
\text { ar, solo, água. } \\
\text { - Modificar a lógica dos serviços de } \\
\text { saúde, dando ênfase à prevenção. } \\
\text { - Promover qualidade de vida, } \\
\text { acesso à educação, saúde, trabalho e } \\
\text { segurança. } \\
\text { - Promover ações de educação para } \\
\text { a saúde e prevenção de doenças e } \\
\text { agravos. }\end{array}$} \\
\hline & $\begin{array}{l}\text { Lógica de } \\
\text { organização dos } \\
\text { serviços } \\
\text { de saúde. }\end{array}$ & $\begin{array}{l}\text { - Taxa de internações em serviços de } \\
\text { saúde. } \\
\text { - Taxa de doenças crônicas. } \\
\text { - Taxa de mortalidade. } \\
\text { - Taxa de morbidade. }\end{array}$ & \\
\hline \multirow[t]{3}{*}{ Pressão } & $\begin{array}{l}\text { Aumento dos } \\
\text { atendimentos em } \\
\text { serviços de saúde } \\
\text { (UBS, clínicas, } \\
\text { ambulatórios). }\end{array}$ & $\begin{array}{l}\text { - Taxa de ocupação hospitalar. } \\
\text { - Taxa de atendimento dos serviços de } \\
\text { saúde. } \\
\text { - Taxa de procedimentos executados. }\end{array}$ & \multirow{3}{*}{$\begin{array}{l}\text { - Promover estilo de vida saudável } \\
\text { que não leve ao adoecimento da } \\
\text { população. } \\
\text { - Diminuir as internações e } \\
\text { necessidade de procedimentos } \\
\text { de serviço de saúde pela atuação } \\
\text { promocional e preventiva. } \\
\text { - Educar profissionais de saúde } \\
\text { para utilizar materiais de forma } \\
\text { sustentável. } \\
\text { - Armazenar, tratar e dispor } \\
\text { adequadamente cada tipo de RSS } \\
\text { conforme sua classificação e manejo. } \\
\text { - Minimizar a geração de RSS e } \\
\text { estimular o reuso e reciclabilidade. }\end{array}$} \\
\hline & $\begin{array}{l}\text { Aumento da } \\
\text { geração de RSS. }\end{array}$ & $\begin{array}{l}\text { - Índice de RSS gerados por tipo de } \\
\text { estabelecimento } \\
\text { - Existência de PGRSS } \\
\text { - Custos com manejo dos RSS. } \\
\text { - Taxa de geração por leito . } \\
\text { - Taxa de geração por procedimento. } \\
\end{array}$ & \\
\hline & $\begin{array}{l}\text { Aumento } \\
\text { na demanda } \\
\text { por correto } \\
\text { tratamento e } \\
\text { disposição final } \\
\text { dos RSS. }\end{array}$ & $\begin{array}{l}\text { - Volume de resíduos gerados e } \\
\text { segregados. } \\
\text { - Quantidade de recipientes de } \\
\text { acondicionamento. } \\
\text { - Existência de programa de capacitação } \\
\text { incluindo a temática sobre resíduos na } \\
\text { educação permanente. } \\
\text { - Custos com destino final. }\end{array}$ & \\
\hline
\end{tabular}

análise do problema estudado. Nesse sentido, reforça-se a posição de autores que, ao avaliar ferramentas disponíveis para proposição de indicadores de saúde e de saúde ambiental em situações de alterações climáticas e saúde ${ }^{24}$ e sustentabilidade $^{25}$, concluem que o Modelo FPSEEA é o mais indicado e completo quando o propósito é perceber uma situação complexa e propor indicadores para a gestão e monitoramento dos processos que determinam e condicionam a saúde.

A sistematização dos elementos (FPSEEA) no Quadro 2 aumenta a visibilidade sobre o fenômeno RSS e permite a proposição de ações e indicadores, o que torna o tema submetido a esta ferramenta de análise, neste caso os RSS, mais facilmente visualizados e compreendidos. Sua importância é ainda maior se considerado que não foram encontrados estudos que utilizassem o Modelo para proposição de indicadores de gerenciamento dos resíduos da saúde.

Destaca-se que é nas fases iniciais de aplicação do Modelo (Força Motriz, Pressão e Situação) que a proposição de intervenções planejadas por meio das Políticas Públicas e dos gestores da saúde e ambiente é fundamental para intervir no desfecho final do problema (Situação, Exposição e Efeito). Por outro lado, nas dimensões exposição e efeito concentram-se as estratégias que podem ser de- 
Quadro 2. Aplicação do Modelo FPSEEA no gerenciamento dos resíduos de serviço de saúde.

\begin{tabular}{|c|c|c|c|}
\hline $\begin{array}{l}\text { Nível do } \\
\text { Modelo }\end{array}$ & Determinantes & Indicadores & Ações \\
\hline \multirow[t]{3}{*}{ Situação } & $\begin{array}{l}\text { Contaminação } \\
\text { ambiental } \\
\text { principalmente } \\
\text { por resíduos } \\
\text { infectantes } \\
\text { e químicos, } \\
\text { alterando } \\
\text { padrões de } \\
\text { qualidade da } \\
\text { água, do Isolo e } \\
\text { do ar. }\end{array}$ & $\begin{array}{l}\text { - Indicadores de qualidade da água e } \\
\text { solo. } \\
\text { - Indicadores de padrão e qualidade do } \\
\text { ar. } \\
\text { - Taxa de poluição ambiental. } \\
\text { - Taxa de emissão de gases na atmosfera. }\end{array}$ & $\begin{array}{l}\text { - Proteger os lençóis freáticos e } \\
\text { mananciais. } \\
\text { - Diminuir a contaminação da água } \\
\text { para consumo. } \\
\text { - Diminuir o uso de água potável } \\
\text { para tratamento de resíduos. } \\
\text { - Reaproveitamento da água. } \\
\text { - Diminuir a emissão de gases } \\
\text { poluentes para promover qualidade } \\
\text { do ar. } \\
\text { - Tratar todo resíduo químico antes } \\
\text { da disposição final. } \\
\text { - Tratar todo resíduo infectante antes } \\
\text { do descarte e/ou disposição final. } \\
\text { - Elaborar e implementar PGRSS. } \\
\text { - Fiscalizar os estabelecimentos } \\
\text { responsáveis pelo tratamento dos } \\
\text { RSS. } \\
\text { - Fiscalizar aterros sanitários e ARIPs. }\end{array}$ \\
\hline & $\begin{array}{l}\text { Risco de } \\
\text { aumento do } \\
\text { número de } \\
\text { acidentes } \\
\text { laborais. }\end{array}$ & $\begin{array}{l}\text { - Taxa de acidentes ocupacionais. } \\
\text { - Dias de afastamento do trabalho } \\
\text { - Custos com tratamento para acidentes } \\
\text { ocupacionais. } \\
\text { - Índice de contaminação por acidente } \\
\text { de trabalho. }\end{array}$ & $\begin{array}{l}\text { - Segregar adequadamente os } \\
\text { resíduos. } \\
\text { - Promover ações de educação } \\
\text { permanente para prevenir e } \\
\text { monitorar as causas dos acidentes. } \\
\text { - Usar adequadamente EPIs. }\end{array}$ \\
\hline & $\begin{array}{l}\text { Risco aumentado } \\
\text { de infecções }\end{array}$ & $\begin{array}{l}\text { - Índice de infecção hospitalar. } \\
\text { - Taxa de infecção cruzada. } \\
\text { - Taxa de reinternação por infecção. }\end{array}$ & $\begin{array}{l}\text { - Inibir formas inadequadas de } \\
\text { manejo. } \\
\text { - Segregação, descarte, } \\
\text { acondicionamento, coleta e } \\
\text { transporte adequado dos resíduos. }\end{array}$ \\
\hline
\end{tabular}

senvolvidas no âmbito das instituições que compõem a rede de serviços de saúde. Nesse estudo são propostos 32 indicadores distribuídos entre os elementos Situação-Exposição-Efeitos do Modelo. Estes podem ser utilizados no monitoramento do processo de gerenciamento dos RSS e, consequentemente, no aprimoramento do Plano de Gerenciamento de Resíduos de Saúde ou nos Planos Municipais de Gestão Integradas de Resíduos, os quais são exigência legal a todas as instituições de saúde geradoras de tais resíduos e ao poder público, respectivamente. Esses elementos podem servir de referência também para a definição de programas de educação permanente específicos.

A complexidade da situação mostra a necessidade de integrar conhecimentos e promover tomadas de decisões no setor saúde que se tornem mais eficientes na mitigação dos impactos dos
RSS na saúde pública (mais diretamente usuários, familiares e trabalhadores na rede de serviços) e nos ecossistemas (qualidade da água, do solo e do ar e a proliferação de vetores $\left.{ }^{26}\right)$. Para o alcance de tal prioridade, a multidisciplinaridade e a interdisciplinaridade são elementos fundamentais para implementar medidas que identifiquem e controlem os riscos que as instituições de saúde (compostas por suas inúmeras particularidades) apresentam para o ambiente e para a vida ${ }^{27}$.

Cabe destacar que as condições de gerenciamento de RSS no Brasil por vezes são inadequadas, potencializando os riscos apontados neste estudo. Assim, considerando este fato e o contexto complexo das instituições de saúde, o Modelo mostra-se relevante na aplicabilidade ao gerenciamento de RSS, especialmente ao considerar que a gestão destes resíduos é fundamental para 
Quadro 2. Aplicação do Modelo FPSEEA no gerenciamento dos resíduos de serviço de saúde.

\begin{tabular}{|c|c|c|c|}
\hline $\begin{array}{l}\text { Nível do } \\
\text { Modelo }\end{array}$ & Determinantes & Indicadores & Ações \\
\hline \multirow[t]{4}{*}{ Exposição } & \begin{tabular}{|l|} 
População \\
exposta a \\
mais fatores \\
determinantes \\
para \\
acometimento da \\
saúde.
\end{tabular} & $\begin{array}{l}\text { - Taxa de ocupação hospitalar. } \\
\text { - Incidência de doenças respiratórias em } \\
\text { crianças e idosos. } \\
\text { - Taxa de internação por problemas } \\
\text { respiratórios. } \\
\text { - Taxa de internação por doenças } \\
\text { crônicas. } \\
\text { - Taxas de reinternação. }\end{array}$ & $\begin{array}{l}\text { - Potencializar política pública } \\
\text { relacionada a qualidade da água, solo } \\
\text { e ar. } \\
\text { - Integrar ações institucionais com as } \\
\text { ações de vigilância epidemiológica e } \\
\text { ambiental. }\end{array}$ \\
\hline & $\begin{array}{l}\text { Água e solo } \\
\text { potencialmente } \\
\text { contaminado } \\
\text { por medicações e } \\
\text { outros produtos } \\
\text { químicos. }\end{array}$ & $\begin{array}{l}\text { - Qualidade da água. } \\
\text { - Taxa de contaminantes presentes na } \\
\text { água. } \\
\text { - Taxa de residências providas de água } \\
\text { potável. } \\
\text { - Taxa de cobertura de coleta seletiva. }\end{array}$ & $\begin{array}{l}\text { - Potencializar política pública de } \\
\text { proteção da água e correto uso para } \\
\text { as atividades. } \\
\text { - Capacitar instituições de saúde } \\
\text { (profissionais e usuários) para } \\
\text { correta segregação. } \\
\text { - Diminuir a contaminação de } \\
\text { mananciais e lençóis freáticos } \\
\text { por meio do correto manejo e } \\
\text { tratamento dos resíduos. } \\
\text { - Diminuir o desperdício da água. }\end{array}$ \\
\hline & $\begin{array}{l}\text { Ar com elevadas } \\
\text { taxas de poluição } \\
\text { e gases poluentes. }\end{array}$ & $\begin{array}{l}\text { - Qualidade do ar. } \\
\text { - Taxa de emissão de gases poluentes. } \\
\text { - Taxa de gases poluentes presentes no ar } \\
\text { ambiente. }\end{array}$ & $\begin{array}{l}\text { - Instituir política de proteção } \\
\text { ambiental para promover a } \\
\text { qualidade do ar para população e } \\
\text { meio ambiente. } \\
\text { - Diminuir a emissão de poluentes } \\
\text { na atmosfera. } \\
\text { - Diminuir a frota de veículos. } \\
\text {-Diminuir a liberação de gases não } \\
\text { tratados. } \\
\text { - Monitorar eficiência dos } \\
\text { incineradores. }\end{array}$ \\
\hline & $\begin{array}{l}\text { Exposição a } \\
\text { patógenos, } \\
\text { resíduos } \\
\text { perigosos } \\
\text { (químicos) em } \\
\text { decorrência do } \\
\text { inadequado } \\
\text { manejo. }\end{array}$ & $\begin{array}{l}\text { - Taxa de doenças ocupacionais. } \\
\text { - Índice de acidentes de trabalho dos } \\
\text { profissionais de saúde. } \\
\text { - Taxa de afastamento do trabalho por } \\
\text { lesões ou doenças ocupacionais. } \\
\text { - Taxa de ocupação hospitalar por } \\
\text { doença respiratória. }\end{array}$ & $\begin{array}{l}\text { - Educar profissionais para manusear } \\
\text { adequadamente resíduos. } \\
\text { - Estimular o uso de EPIs e EPCs. } \\
\text { - Disponibilizar EPIs de acordo com } \\
\text { o nível de risco. } \\
\text { - Promover medidas de vigilância e } \\
\text { de atenção à saúde. } \\
\text { - Estabelecer fluxo de notificação de } \\
\text { doenças e agravos. } \\
\text { - Estabelecer medidas de prevenção } \\
\text { das doenças. } \\
\text { - Elaborar e implementar o PGRSS. } \\
\text { - Atender todos os dispositivos legais } \\
\text { existentes. }\end{array}$ \\
\hline
\end{tabular}

continua

a proteção e a promoção do meio ambiente e da saúde pública.

Há que se considerar ainda que os RSS possuem características diferentes das demais categorias de resíduos por conterem grande quantidade de material biológico resultante da assistência direta à saúde humana e de substâncias químicas como desinfetantes, antibióticos e medicamentos, dos quais decorrem os riscos biológicos e químicos ${ }^{26}$. Tais riscos também podem 
Quadro 2. Aplicação do Modelo FPSEEA no gerenciamento dos resíduos de serviço de saúde.

\begin{tabular}{|c|c|c|c|}
\hline $\begin{array}{l}\text { Nível do } \\
\text { Modelo }\end{array}$ & Determinantes & Indicadores & Ações \\
\hline \multirow[t]{3}{*}{ Efeito } & $\begin{array}{l}\text { Doenças } \\
\text { infecciosas } \\
\text { transmissíveis } \\
\text { por material } \\
\text { perfurocortante. }\end{array}$ & \multirow{3}{*}{$\begin{array}{l}\text { - Taxa de acidentes com } \\
\text { perfurocortantes. } \\
\text { - Taxa de uso de retrovirais por } \\
\text { profissionais de saúde acidentados. } \\
\text { - Taxa de ocupação hospitalar por } \\
\text { doenças infecciosas. } \\
\text { - Taxa de surtos por doenças diarreicas. } \\
\text { - Taxa de doenças transmitidas por } \\
\text { veiculação dos elementos naturais } \\
\text { potencialmente contaminados e } \\
\text { alterados. }\end{array}$} & \multirow{3}{*}{$\begin{array}{l}\text { - Promover educação permanente } \\
\text { nas instituições com a temática de } \\
\text { biossegurança e resíduos de serviço } \\
\text { de saúde. } \\
\text { - Promover tratamento adequado } \\
\text { para o profissional acidentado. } \\
\text { - Promover adequadas formas } \\
\text { e processos de armazenamento, } \\
\text { tratamento e destinação dos } \\
\text { resíduos. }\end{array}$} \\
\hline & $\begin{array}{l}\text { Aumento da taxa } \\
\text { de infecção. }\end{array}$ & & \\
\hline & $\begin{array}{l}\text { IAumento } \\
\text { das doenças } \\
\text { e problemas } \\
\text { de saúde } \\
\text { ocasionados por } \\
\text { componente na } \\
\text { água, solo e ar. }\end{array}$ & & \\
\hline
\end{tabular}

Fonte: elaborado pelos autores (2015) com base em Pinto et al. ${ }^{14}$.

ser minimizados por ações relacionadas à "exposição” conforme apresentado no Quadro 2.

Ainda sobre os riscos biológicos, vale destaque que os patógenos Mycobacterium tuberculosis, o vírus da hepatite A e B, Escherichia coli e Staphylococcus aureus apresentam capacidade de sobreviver ou resistir no meio ambiente intra ou extramuros dos serviços de saúde, quando não adequadamente tratados. A magnitude desses riscos biológicos quanto à possibilidade do acometimento da saúde humana e ambiental tem relação direta com o gerenciamento dos $\mathrm{RSS}^{28}$, que, quando inadequado aumenta a probabilidade de ocorrência de danos à saúde e ao ambiente. A proteção do ambiente está ligada mais diretamente aos elementos "pressão" e "situação", o que implica ações não apenas no âmbito das instituições de saúde, mas compartilha a responsabilidade com o poder público, especialmente ao definir os planos municipais para gestão integrada dos resíduos. Significa que são ações a serem desenvolvidas no contexto geral da sociedade.

Desta forma, o gerenciamento é imprescindível, pois tem como objetivo reduzir as ameaças que os RSS apresentam, ou seja, a gestão adequada visa reduzir os riscos sanitários e ambientais, assim como a promoção da qualidade de vida e da saúde coletiva e o desenvolvimento sustentável ${ }^{29}$.

No caso do gerenciamento de RSS, conforme demonstrado no Quadro 2, a aplicação do Modelo permite subsidiar ações para a problemática com vistas à preservação, proteção e promoção da saúde ambiental, meio ambiente e saúde pública. Neste sentido, incorporar indicadores ambientais no Modelo FPSEEA junto com os indi- cadores de saúde, é possibilitar a concepção ampliada da saúde, superando a visão fragmentada do processo saúde-doença ${ }^{16}$ Especificamente no caso dos RSS, a aplicação do Modelo pode ser representada conforme a Figura 2.

A Figura 2 apresenta esquematicamente a implicação da geração de resíduos de serviço de saúde em cada elemento do Modelo. Dado o contexto econômico, social e cultural que produzem pressões no estado de saúde da população, esta pressão resulta em aumento dos atendimentos em serviço de saúde e, consequentemente, na geração de RSS. Assim, a situação resultante pode ser percebida por meio de contaminação ambiental, de acidentes laborais e de infecções. Esta situação expõe a população a fatores que determinam acometimentos no estado de saúde, elementos naturais contaminados e poluídos, exposição a patógenos e resíduos perigosos. E por fim, o efeito de toda essa rede será as alterações no estado de saúde com produção de doença, tendo impactos na Saúde Pública. Inverter essa situação implica em utilizar os indicadores no monitoramento dos contextos de geração de resíduos e implementar ações que possam produzir efeitos benéficos para a saúde.

\section{Considerações finais}

O gerenciamento de resíduos de serviços de saúde é complexo, uma vez que envolve resíduos perigosos gerados de forma crescente na assistência à saúde da população. Seu manejo adequado representa minimização dos riscos decorrentes e, 


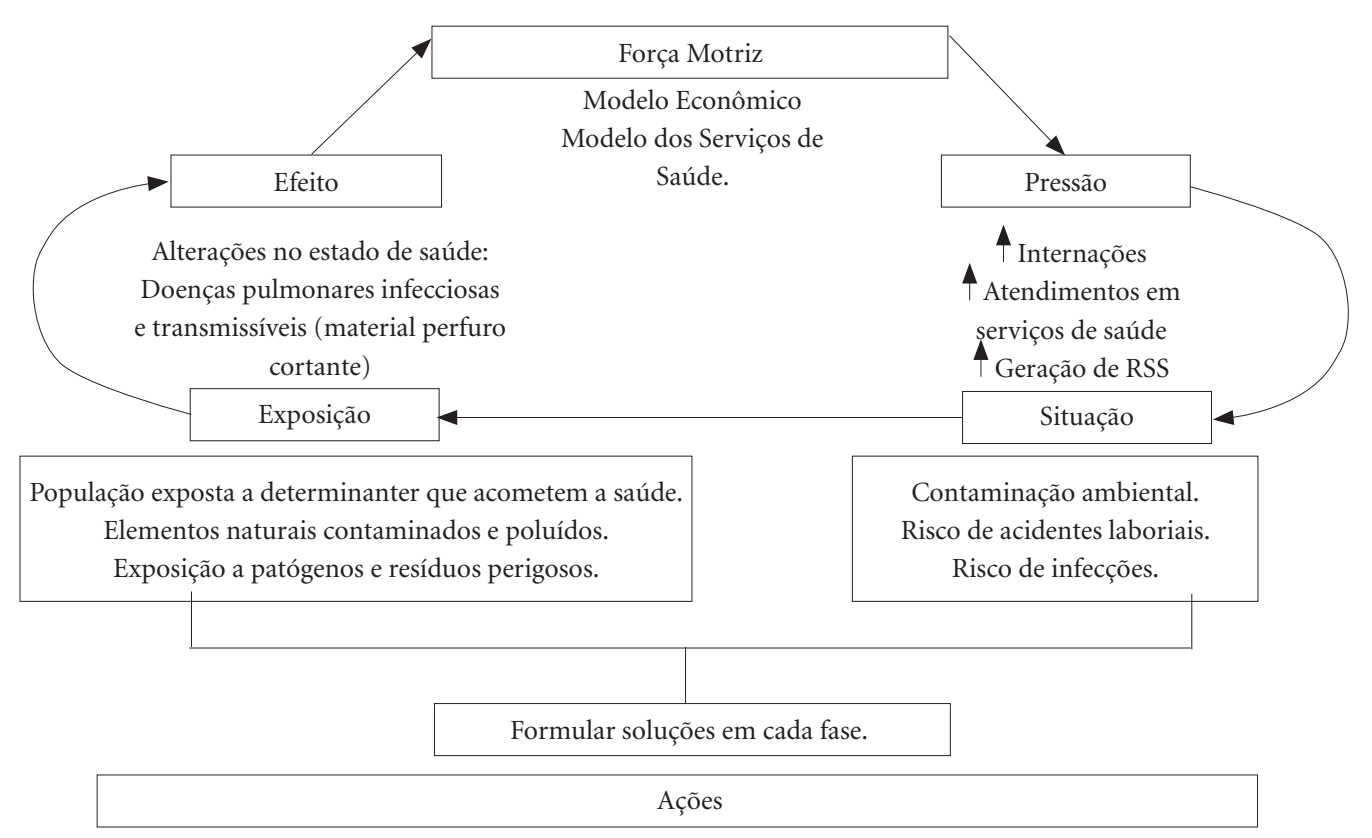

Figura 2. aplicação do Modelo FPSEEA ao gerenciamento de RSS.

Fonte: elaborado pelos autores (2015).

ao contrário, quando manejados de forma incorreta, acometem a saúde física e ambiental.

O gerenciamento adequado, por sua vez, exige a análise de cada situação específica onde esses são gerados e a proposição de indicadores que possam ser utilizados no monitoramento do processo e do controle dos riscos a ele associados. O Modelo FPSEEA é indicado ao gerenciamento de RSS por permitir a visualização e análise do contexto onde os resíduos são gerados, bem como a proposição de indicadores e das ações com potencialidade para o alcance dos resultados almejados. Dito em outras palavras, o Modelo FPSEEA possibilita intervir nos problemas a serem monitorizados e solucionados, além de ter alcance na integração das ações em diferentes níveis das forças que acometem a saúde e o meio ambiente a $18,25,30,31^{\text {. }}$.

Sua utilidade é ainda maior se considerado que a Saúde Ambiental é um campo novo, para onde emergem diferentes áreas do conhecimento e, como tal, carece de indicadores específicos que possam auxiliar na elucidação das complexas relações entre saúde e ambiente, bem como no estabelecimento dos nexos causais entre essas duas áreas.

A preservação ambiental e a promoção da qualidade de vida dependem de ações profissionais e de gestores de saúde e do ambiente. Uma visão com foco na prevenção e planejamento de ações é fundamental para propiciar melhores condições de trabalho, estudo, crescimento, desenvolvimento dos sujeitos e das comunidades e proteção de coletividades. No caso do gerenciamento de resíduos de serviço de saúde a aplicação do Modelo mostrou-se útil na definição de indicadores e na proposição de ações intersetoriais, além de possibilitar a percepção mais detalhada do que precisa ser levado em conta em programas de educação permanente que capacitem os profissionais e gestores a utilizar ferramentas favorecedoras da tomada de decisões para alcance de melhor qualidade de vida da sociedade e preservação da vida no planeta. 


\section{Colaboradores}

NLR Stedile trabalhou na concepção, delineamento e análise dos dados, revisão crítica e aprovação da versão a ser publicada. VE Schneider trabalhou na revisão crítica e aprovação da versão final. MW Nunes trabalhou na redação do artigo, na pesquisa, na metodologia, na análise dos dados. AC Kappes trabalhou na análise dos dados e na revisão crítica.

\section{Referências}

1. Brasil. Ministério da Saúde (MS). Saúde ambiental: guia básico para construção de indicadores. Brasília: MS; 2011.

2. Schneider VE, Stedile NLR, organizadores. Resíduos de serviços de saúde: um olhar interdisciplinar sobre o fenômeno. 3a ed. Caxias do Sul: Educs; 2015.

3. Brasil. Ministério da Saúde (MS). Subsídios para construção da Política Nacional de Saúde Ambiental. Brasília: Editora do Ministério da Saúde; 2007.

4. Brasil. Lei $\mathrm{n}^{\circ} 12.305$, de 2 de agosto de 2010. Institui a Política Nacional de Resíduos Sólidos. Diário Oficial da União 2010; 2 ago.

5. Brasil. Conselho Nacional de Meio Ambiente. Resolução no 358, de 29 de abril de 2005. Dispõe sobre o tratamento e a disposição final dos resíduos dos serviços de saúde e dá outras providências. Diário Oficial da União 2005; 4 mai.

6. Siqueira MM, Moraes MS. Saúde coletiva, resíduos sólidos urbanos e catadores de lixo. Cien Saude Colet 2009; 14(6):2115-2122.

7. Mathur V, Dwivedi S, Hassan MA, Misra RP. Knowledge, Attitude, and Practices about Biomedical Waste Management among Healthcare Personnel: A Cross-sectional Study. Indian J Community Med 2011; 36(2):143-145.

8. Babanyara YY, Ibrahim DB, Garba T, Bogoro AG, Abubakar, MY. Poor Medical Waste Management (MWM) practices and its risks to human health and the environment: a literature review. International Journal of Environmental, Earth Science and Engineering 2013; 11(7):1-8

9. Organización Pan-Americana de la Salud (OPAS). Indicadores básicos de salud ambiental para la región de la frontera México - Estados Unidos. Washington: OPAS; 2001. [Documento conceptual].

10. Organização Pan-Americana da Saúde (OPAS), Rede Interagencial de Informações para a Saúde (RIPSA). Indicadores básicos para a saúde no Brasil: conceitos e aplicações. Brasília: OPAS/RIPSA; 2002.

11. Corvalan C, Briggs D, Zielhuis G. Decision-making in environmental health: from evidence to action. London: Spon; 2000.

12. Oliveira MLBC, Faria SC. Aplicação do modelo FPSEEA na construção de indicadores de saúde ambiental. In: Philippi AJ, Malheiros TF, editores. Indicadores de sustentabilidade e gestão ambiental. Barueri, SP: Manole; 2012. p. 445-471.

13. Oliveira MLC, Faria SC. Indicadores de saúde ambiental na formulação e avaliação de políticas de desenvolvimento sustentável. Revista Brasileira de Ciências Ambientais 2008; 11:16-22.

14. Pinto MA, Peres F, Moreira JC. Utilização do modelo FPEEA (OMS) para a análise dos riscos relacionados ao uso de agrotóxicos em atividades agrícolas do estado do Rio de Janeiro. Cien Saude Colet 2012; 17(6):15431555.

15. Oliveira MLBC. Possibilidades de aplicação do modelo FPSEEA/OMS na construção de indicadores de saúde ambiental - DF [dissertação]. Brasília: Universidade Católica de Brasília; 2007. 
16. Sobral A, Freitas CM. Modelo de Organização de Indicadores para Operacionalização dos Determinantes Socioambientais da Saúde. Saúde Soc 2010; 19(1):3547.

17. World Health Organization (WHO). Environmental health indicators for Europe - A pilot indicator-based report. Copenhagen: WHO; 2004.

18. Briggs D. Environmental health indicators: frameworks and methodologies. Protection of the Human Environment - Occupational and environmental health series. Geneva: World Health Organization (WHO), Nene Center for research University College Northampton; 1999.

19. Briggs D. Making a difference: Indicators to improve children's environmental health. Geneva: World Health Organization (WHO); 2003.

20. Franco Netto G, Freitas CM, Andahur JP, Pedroso MM, Rohlfs DB. Impactos socioambientais na situação de saúde da população brasileira: Estudo de indicadores relacionados ao saneamento ambiental inadequado. Tempus. Actas em Saúde Coletiva 2009; 4(4):53-71.

21. Gil AC. Como elaborar projetos de pesquisa. $3^{\text {a }}$ ed. São Paulo: Atlas; 1991.

22. Bardin L. Análise de conteúdo. São Paulo: Edições 70; 2011.

23. Moraes R. Análise de conteúdo. Revista educação 1999; 22(37):7-32.

24. Hambling T, Philip W, Slaney D. A review of frameworks for developing environmental health indicators for climate change and health. International journal of environmental research and public health 2011; 8(7):2854-2875.

25. Waheed B, Khan F, Veitch B. Linkage-based frameworks for sustainability assessment: making a case for driving force-pressure-state-exposure-effect-action (DPSEEA) frameworks. Sustainability 2009; 1(3):441-463.
26. Garcia LP, Ramos BGZ. Health services waste management: a biosafety issue. Cadernos de Saude Publica 2004; 20(3):744-752.

27. Kligerman DC, Vilela H, Cardoso TAO, Cohen SC, Sousa D, Rovere E. Sistemas de indicadores de saúde e ambiente em instituições de saúde. Cien Saude Colet 2007; 12(1):199-211.

28. Silva ACN, Bernardes RS, Moraes LRS, Reis JDP. Critérios adotados para seleção de indicadores de contaminação ambiental relacionados aos resíduos dos serviços de saúde: uma proposta de avaliação. Cad Saude Publica 2002; 18(5):1401-1409.

29. Brasil. Ministério da Saúde (MS). Manual de gerenciamento de resíduos de serviços de saúde. Brasília: MS; 2006. (Série A. Normas e Manuais Técnicos).

30. Füssel HM. Adaptation planning for climate change: concepts, assessment approaches, and key lessons. Sustainability science 2007; 2(2):265-275.

31. Corvalán CF, Kjellstrom T, Smith KR. Health, environment and sustainable development: identifying links and indicators to promote action. Epidemiology-Baltimore 1999; 10(5):656.

Artigo apresentado em 16/12/2015

Aprovado em 20/10/2016

Versão final apresentada em 22/10/2016 